\title{
Efficient delivery of Notch I siRNA to SKOV3 cells by cationic cholesterol derivative-based liposome
}

This article was published in the following Dove Press journal:

International Journal of Nanomedicine

20 October 2016

Number of times this article has been viewed

\author{
Yun-Chun Zhao' \\ Li Zhang ${ }^{2}$ \\ Shi-Sen Feng ${ }^{3}$ \\ Lu Hong ${ }^{3}$ \\ Hai-Li Zheng ${ }^{3}$ \\ Li-Li Chen ${ }^{4}$ \\ Xiao-Ling Zheng' \\ Yi-Qing $Y e^{\prime}$ \\ Meng-Dan Zhao' \\ Wen-Xi Wang ${ }^{3}$ \\ Cai-Hong Zheng'
}

'Pharmacy Department, Women's Hospital, ${ }^{2}$ Pharmacy Department,

The Second Affiliated Hospital,

School of Medicine, Zhejiang

University, Hangzhou, People's

Republic of China; ${ }^{3}$ Department of

Pharmaceutic Preparation, College

of Pharmaceutical Science, Zhejiang

University of Technology, Hangzhou,

${ }^{4}$ Department of Gynecologic

Oncology, Women's Hospital, School

of Medicine, Zhejiang University,

Hangzhou, People's Republic of China

Correspondence: Cai-Hong Zheng

Women's Hospital, School of Medicine,

Zhejiang University, I Xueshi Road,

Hangzhou 310006, People's Republic

of China

Tel $+8657 \mid 89991730$

Fax +86 57| 8999 |730

Email chzheng@zju.edu.cn

Wen-Xi Wang

College of Pharmaceutical

Science, Zhejiang University of

Technology, I 8 Chaowang Road,

Hangzhou 310014, People's Republic

of China

Tel +8657188320320

Fax +86 57I 88320320

Email yjw@zjut.edu.cn

\begin{abstract}
A novel cationic cholesterol derivative-based small interfering RNA (siRNA) interference strategy was suggested to inhibit Notch1 activation in SKOV3 cells for the gene therapy of ovarian cancer. The cationic cholesterol derivative, $N$-(cholesterylhemisuccinoylamino-3-propyl)- $N$, $N$-dimethylamine (DMAPA-chems) liposome, was incubated with siRNA at different nitrogen-to-phosphate ratios to form stabilized, near-spherical siRNA/DMAPAchems nanoparticles with sizes of 100-200 nm and zeta potentials of 40-50 mV. The siRNA/ DMAPA-chems nanoparticles protected siRNA from nuclease degradation in $25 \%$ fetal bovine serum. The nanoparticles exhibited high cell uptake and Notch1 gene knockdown efficiency in SKOV3 cells at an nitrogen-to-phosphate ratio of 100 and an siRNA concentration of $50 \mathrm{nM}$. They also inhibited the growth and promoted the apoptosis of SKOV3 cells. These results may provide the potential for using cationic cholesterol derivatives as efficient nonviral siRNA carriers for the suppression of Notch1 activation in ovarian cancer cells.
\end{abstract}

Keywords: siRNA, cationic cholesterol derivative, Notch1, ovarian cancer cells

\section{Introduction}

Epithelial ovarian cancer is the most lethal gynecological malignancy. ${ }^{1}$ Because of its high incidence and mortality rates, ovarian cancer is a lethal and underrecognized detriment to women's health all over the world. Despite the improvement in therapy, patients with advanced ovarian cancer have a low 5-year survival rate of only $10 \%-20 \%{ }^{2}$ Traditionally, ovarian cancer is treated with platinum-based chemotherapy followed by surgical tumor debulking; however, cisplatin resistance is a major obstacle to the successful treatment of ovarian cancer. Several mechanisms have been reported to be related to the development of cisplatin resistance, including reduced uptake, enhanced efflux, increased inactivation, and evasion of apoptosis. ${ }^{3}$ Thus, developing a novel therapeutic approach is extremely important for the treatment of ovarian cancer.

In recent years, RNA interference approaches, especially utilizing small interfering RNA (siRNA), have received considerable attention in the treatment of ovarian cancer. ${ }^{46}$ The siRNA approach, as a therapeutic method of blocking neovascularization, was reported to have incomparable long-term effects as well as safety effects. ${ }^{7-9}$ The Notch signaling pathway plays a pivotal role in cellular development, proliferation, differentiation, and apoptosis. It has been shown to be involved in the carcinogenesis, progression, invasion, and neovascularization of many solid tumors. ${ }^{10}$ Notch 1 can function as a tumor suppressor or as an oncogene depending on the cell type and context. It acts as a tumor inhibitor in murine skin tumors ${ }^{11}$ and non-small-cell 
lung cancer. ${ }^{12}$ In contrast, it acts as an oncogene in many other kinds of tumors, such as renal cancer, pancreatic cancer, cervical cancer, breast cancer, and prostate cancer. ${ }^{13}$ Notch1 is considered to act as an oncogene in ovarian cancer, and high Notch1 protein expression was discovered in ovarian cancers and corresponding ovarian carcinoma cell lines, including SKOV3 and A2780 cells. ${ }^{14,15}$ Notch1 expression has been reported to correlate to tumor differentiation status in ovarian cancer. ${ }^{16,17}$

Although siRNA is small in size, siRNA transportation across the cytomembrane is hindered by its hydrophility and negative electric charge and is easily eliminated during in vivo circulation before arrival at the target site. ${ }^{18}$ Therefore, effective siRNA-mediated therapy relies heavily on the improvement of siRNA delivery carriers with high efficiency of transfection, the ability to easily escape the endosome, and minimal cytotoxicity. ${ }^{19}$ Nonviral gene delivery vectors, particularly cationic polymers (eg, chitosan, polyethyleneimine, and polylysine peptides), liposomes, and micelles, have attracted increasing attention in gene therapeutic strategy. ${ }^{20-23}$ A typical cationic lipid generally consists of three parts: cationic head groups, linkers/linkages, and hydrophobic lipid tails. However, the exact combination of these three parts to achieve lower cytotoxicity and higher transfection performance is still a major challenge as it relies on the good understanding of the structure-activity relationships of lipid carriers. Compared with cationic lipid-based liposomes, the cationic cholesterol derivative-based liposomes are cheaper to get and also have lower cytotoxicity. ${ }^{24,25}$ Liu et $\mathrm{al}^{26}$ used nanoparticles based on hyper-forked cationic polysaccharide derivatives to investigate the delivery of siRNA into human retinal pigment epithelial (hRPE) cells to silence NF- $\kappa B$ gene expression. They found that a 3-(dimethylamino)1-propylamine-conjugated glycogen (DMAPA-Glyp) derivative could condense siRNA efficiently owing to its higher branching structure. The DMAPA-Glyp derivative showed less toxicity to hRPE cells and facilitated better protection of siRNA from degradation by nucleases. Due to their small sizes and high zeta potentials, the DMAPA-Glyp/siRNA nanoparticles effectively transferred siRNA to the hRPE cells. This led to significant suppression of the expression of NF- $\kappa$ B p65 mRNA and protein in those cells transfected with DMAPAGlyp/siRNA nanoparticles. In a study by Hattori et al, ${ }^{27}$ they synthesized a new cationic cholesteryl triamine $(\mathrm{OH}-\mathrm{N}-\mathrm{Chol}$, (3S)-N-(2-(2-(2-hydroxyethylamino) ethylamino) ethyl) cholesteryl -3-carboxamide ) with an ethylenimine extension and prepared cationic nanoparticles (NP-N) composed of OH-N-Chol and Tween 80 which could form an siRNA/NP-N nanoplex $200 \mathrm{~nm}$ in size on vortex mixing. The NP-N nanoplex was internalized via macropinocytosis-mediated endocytosis and produced a high level of gene knockdown. We recently reported that $N$-(cholesterylhemisuccinoyl-amino3-propyl)- $N, N$-dimethylamine (DMAPA-chems) liposomes showed good blood compatibility and low cytotoxicity and could efficiently deliver plasmid DNA in vitro. ${ }^{28}$ However, whether DMAPA-chems cationic cholesterol derivatives can effectively deliver siRNA into SKOV3 cells and efficiently knockdown the target gene have not yet been elucidated.

In this study, DMAPA-chems liposomes were successfully synthesized, and the interference strategy based on cationic cholesterol derivative liposomes was used to develop an excellent siRNA delivery system for efficient cellular uptake and knockdown of the Notch1 gene in SKOV3 human ovarian cancer cells. The siRNA/DMAPA-chems complexes were estimated for cytotoxicity, stability, special gene silencing efficiency, cell proliferation, and apoptosis at different nitrogen-to-phosphate $(\mathrm{N} / \mathrm{P})$ ratios and at different doses of siRNA in SKOV3 cells.

\section{Materials and methods Materials}

$N, N$-dimethyl propylene diamine (DMAPA) was purchased from Aladdin (Shanghai, People's Republic of China), $N$-hydroxysuccinimide (NHS) was supplied by SigmaAldrich (St Louis, MO, USA), cholesterol was procured from Sinopharm (Shanghai, People's Republic of China), soybean phospholipid S100 was bought from Lipoid (Ludwigshafen, Germany), polymerase chain reaction (PCR) primers were supplied by Generay (Shanghai, People's Republic of China), and negative control (NC) siRNA and Notch1-homo-siRNA or a green fluorescent agent FAM at the $3^{\prime}$ end of the sense strand or antisense strand were procured from Genepharma (Shanghai, People's Republic of China). Lipofectamine 2000 and Trizol were obtained from Thermo Fisher Scientific (Waltham, MA, USA). PrimeScript RT Reagent Kit and SYBR Premix Ex Tap Reagent Kit were obtained from TakaRa (Dalian, People's Republic of China). Cell Counting Kit-8 (CCK-8) was obtained from Dojindo (Tokyo, Japan). Annexin V-fluorescein isothiocyanate (FITC) apoptosis detection kit was purchased from Multi Sciences (Shanghai, People's Republic of China). HRP-conjugated goat anti-mouse $\operatorname{IgG}(\mathrm{H}+\mathrm{L})$, sodium dodecyl sulfate polyacrylamide gel electrophoresis (SDS-PAGE) sample loading buffer, and anti- $\beta$-actin antibody were procured from Auragene (Hunan, People's Republic of China), and anti-Notch1 antibody was provided by Abcam (Cambridge, MA, USA). The SKOV3 cells were obtained 
from the American Type Culture Collection (ATCC, HTB-77). McCoy's 5A, trypsin/ethylenediaminetetraacetic acid (EDTA), and phosphate buffer solution (PBS) were bought from Genom (Hangzhou, People's Republic of China). Fetal bovine serum (FBS) was purchased from Thermo Fisher Scientific.

\section{Preparation of the siRNA/DMAPA-chems nanoparticles}

A novel kind of cholesterol cationic lipids (DMAPA-chems) was designed and synthesized, in which natural cholesterol was employed as a hydrophobic block, and DMAPA served as a kind of cationic head group; the hydrophobes and head groups were connected through flexible 1,4-succinic anhydride linkers with biodegradable carbonate ester linkage bonds. The synthesis of DMAPA-chems is accomplished in several simple and easy steps as previously described. ${ }^{28}$ Forty milliliters of triethylamine was added to a mixture of $5.0 \mathrm{~g}$ commercially available cholesteryl and $4.0 \mathrm{~g}$ succinic anhydride in $150 \mathrm{~mL}$ tetrahydrofuran (THF) at $80^{\circ} \mathrm{C}$. After 48 hours, the solvent was removed by vacuum distillation at $40^{\circ} \mathrm{C}$. The reactant was dissolved with $60 \mathrm{~mL}$ ethanol. The combined organic phase was extracted with $100 \mathrm{~mL}$ deionized water and recrystallized twice from acetone to obtain the cholesteryl hemisuccinate. The obtained cholesteryl hemisuccinate was added to NHS to excite the cholesteryl hemisuccinate to a semistable aminereactive activated NHS-ester. DMAPA-chems was prepared by reacting $0.5 \mathrm{~g}$ activated ester with $10 \mathrm{~mL}$ DMAPA $(1.2 \%$ dichloromethane $\left[\mathrm{CH}_{2} \mathrm{Cl}_{2}\right]$ solution) in $20 \mathrm{~mL} \mathrm{CH}_{2} \mathrm{Cl}_{2}$ on ice for 1 hour, then precipitated in cold isopropanol, purified by cold ether, and lyophilized.

To produce liposomes, $19 \mathrm{mg}$ DMAPA-chems and $50 \mathrm{mg}$ phospholipid S100 were combined using the film dispersion method. The cationic cholesterol derivative DMAPA-chems liposomes were at a concentration of $3.8 \mathrm{mg} / \mathrm{mL}$. The lyophilized siRNA was dissolved into RNase-free water, forming a $20 \mu \mathrm{M}$ stock solution. The siRNA stock solution in part was diluted in Opti-MEM reduced serum media (Thermo Fisher Scientific) of $200 \mu \mathrm{L}$ and incubated for 5 minutes at room temperature. Then, the DMAPA-chems solution was added at different N/P ratios. The mixtures were gently agitated and then incubated at room temperature for 20 minutes before use. Nanoparticles containing FAM-siRNA were obtained in the same way in the darkroom.

\section{Particle size, zeta potential, and morphology}

The particle size and zeta potential of siRNA/DMAPA-chems nanoparticles at different $\mathrm{N} / \mathrm{P}$ ratios were measured using dynamic light scattering with a laser Doppler anemometry (Delsa Nano C A53878, Beckman Coulter, Brea, CA, USA). The diameter and zeta potential values were measured in triplicate. The morphology of siRNA/DMAPA-chems complexes $(\mathrm{N} / \mathrm{P}=100)$ was observed using transmission electron microscopy (JEM-1230, JEOL, Tokyo, Japan), and the nanoparticles were negatively stained using $2 \mathrm{wt} \%$ aqueous uranyl acetate.

\section{Agarose gel electrophoresis}

Electrophoresis tests were carried out to observe the condensation ability of the cationic cholesterol derivatives to siRNA. Ten microliters of the siRNA/DMAPA-chems nanoparticles with various N/P ratios in the range of $2-80$ and naked siRNA were loaded onto $1.5 \%$ agarose gels containing $1 \mu \mathrm{L}$ of $2 \%$ bromophenol blue loading buffer and run with Tris/Borate/ EDTA buffer at $100 \mathrm{~V}$ for 20 minutes. siRNA retardation was then detected and photographed with a gel document imaging system (Gel DocTMXR+, Bio-Rad Laboratories Inc., Hercules, CA, USA).

\section{Stability analysis}

To estimate the protective property of the cationic cholesterol derivative against siRNA degradation, the siRNA/DMAPAchems nanoparticles $(\mathrm{N} / \mathrm{P}=50)$ containing $1 \mu \mathrm{g}$ siRNA were incubated with $25 \% \mathrm{FBS}$ and $0.1 \mathrm{mg} / \mathrm{mL}$ RNase A at $37^{\circ} \mathrm{C}$ for 24 hours. At the same time, naked siRNA was incubated with $25 \%$ FBS (the NC). Samples were incubated for 1 hour with excess heparin solution at a siRNA/heparin weight ratio of 1:5 to ensure siRNA released from the nanoparticles completely. ${ }^{26}$ The samples were assessed using an agarose gel document imaging system, as described earlier, and the integrity of siRNA was detected.

\section{Cell culture}

SKOV3 cells were cultured in McCoy's 5A, which was supplemented with $10 \% \mathrm{FBS}, 100 \mathrm{U} / \mathrm{mL}$ penicillin, and $100 \mu \mathrm{g} / \mathrm{mL}$ streptomycin; the cells were then incubated at $37^{\circ} \mathrm{C}$ in a $5 \% \mathrm{CO}_{2}$ atmosphere. After rinsing with $\mathrm{PBS}$, the cells were detached using trypsin/EDTA and then resuspended in a fresh medium for subsequent experiments.

\section{Cytotoxicity assay}

To evaluate the cytotoxicity of the cationic cholesterol derivatives and the siRNA/DMAPA-chems nanoparticles, a CCK-8 assay was performed. SKOV3 cells $\left(6 \times 10^{3}\right.$ per well) were plated into 96-well plates and incubated in a humidified incubator $\left(37^{\circ} \mathrm{C}, 5 \% \mathrm{CO}_{2}\right)$ for 24 hours for adherence. The medium 
was then replaced with $100 \mu \mathrm{L}$ of fresh medium containing DMAPA-chems liposomes at different concentrations ranging from 10 to $800 \mu \mathrm{g} / \mathrm{mL}$. siRNA/DMAPA-chems nanoparticles with various N/P ratios from 1 to 120 were added for the cytotoxicity assay. After the cells were incubated for 24,48 , and 72 hours, $10 \mu \mathrm{L}$ of the CCK-8 solution was added to each well of the experiment plate, and incubated for 1.5 hours. The absorbance was measured at $450 \mathrm{~nm}$ using a microplate reader (Varioskan Frash, Thermo Fisher Scientific). All experiments were repeated in quintuplicate.

\section{Quantification of cellular uptake}

Flow cytometry analysis was used to analyze the cellular uptake of siRNA/DMAPA-chems nanoparticles. SKOV3 cells were plated into six-well plates and incubated in complete McCoy's 5A until they reached 50\% confluence. The cells were cultured with FAM-siRNA/DMAPA-chems complexes $(\mathrm{N} / \mathrm{P}=20,40,60,80,100$, or 120$)$ at a FAM-siRNA dose of $50 \mathrm{nM}$. After incubation about 5 hours, the medium was discarded and the cells were rinsed twice with cold PBS twice. The cells were then trypsinized, washed with cold PBS three times, and subjected to analysis using a flow cytometer (BD FACSVerse, BD Biosciences, San Jose, CA, USA).

\section{Quantitative real-time PCR (qRT-PCR) analysis}

SKOV3 cells were seeded in six-well plates $\left(2 \times 10^{5}\right.$ cells/ well), incubated for 24 hours, then transfected with Notch1 siRNA/DMAPA-chems complexes $(\mathrm{N} / \mathrm{P}=80,100$, or 120$)$ at an siRNA dose of $50 \mathrm{nM}$. In addition, different siRNA doses (30, 50, 80, or $100 \mathrm{nM})$ of Notch1 siRNA/DMAPA-chems complexes at a fixed N/P ratio of 100 were also investigated. SKOV3 cells without any disposure were used as the control, while SKOV3 cells cultured with the nonspecific siRNA/ Lipo complex were grouped as the NC. Notch1 siRNA/lipofectamine 2000 served as a positive control. The cells were then cultured at $37^{\circ} \mathrm{C}$ for 5 hours and rinsed with PBS solution. The medium was substituted with fresh serum-supplemented medium and further cultured at $37^{\circ} \mathrm{C}$. After incubation for 48 hours, Trizol reagent was used to extract total RNA from SKOV3 cells. qRT-PCR was performed as described in the SYBR Premix Ex Tap kit procedure instructions: denaturation, 1 cycle at $95^{\circ} \mathrm{C}$ for 2 minutes; amplification, 40 cycles at $95^{\circ} \mathrm{C}$ for 15 seconds, $60^{\circ} \mathrm{C}$ for 30 seconds; final extension, 70 cycles at $95^{\circ} \mathrm{C}$ for 1 minute, $60^{\circ} \mathrm{C}$ for 1 minute, $60^{\circ} \mathrm{C}$ to $95^{\circ} \mathrm{C}(10 \mathrm{~s} /$ cycle, $0.5^{\circ} \mathrm{C} /$ cycle), using a sequence detection system (AB VIIA 7DX, Thermo Fisher Scientific). A value of $2^{-\Delta \Delta C t}$ was figured out to estimate Notch1 mRNA expression.

\section{Western blot analysis}

SKOV3 cells were seeded into six-well plates and incubated for 24 hours, which was followed by incubation with the Notch1 siRNA/DMAPA-chems nanoparticles (N/P =100) at an siRNA dose of $50 \mathrm{nM}$ for 5 hours. Afterward, the medium was replaced with fresh McCoy's 5A supplemented with $10 \%$ FBS. After an additional 24, 48, and 72 hours of incubation, the medium was discarded. After washing with PBS twice, the cells were collected for protein extraction. To each well, $150 \mu \mathrm{L}$ of radioimmunoprecipitation assay lysis buffer was added and the six-well plates were shaken on an ice-bath for 45 minutes. The lysate was gathered and centrifuged at $14,000 \times g$ at $4^{\circ} \mathrm{C}$ for 10 minutes. Forty micrograms of total protein per lysate were separated on an $8 \%$ SDS-PAGE gel and then transferred to a nitrocellulose membrane. The membrane was immersed in 5\% nonfat milk Tris buffer for 1 hour and then cultured overnight with an anti-Notch1 antibody. An anti- $\beta$-actin antibody acted as the Western blot loading control. The membrane was incubated with a secondary antibody for 1 hour. After rinsing with Tris buffer for 30 minutes, a near-infrared two-color laser scanner imaging system (Image Quant LAS 4000 mini, GE Healthcare Life Sciences, Chicago, IL, USA) was used to scan and photograph the membrane.

\section{Cell proliferation assay}

CCK-8 assay was also used to observe the cell proliferation of the Notch1 siRNA/DMAPA-chems at various N/P ratios $(80,100$, and 120), with NC siRNA/DMAPA-chems $(\mathrm{N} / \mathrm{P}=80,100$, or 120$)$ as a NC. SKOV3 cells were plated into 96-well plates at an initial density of $6 \times 10^{3}$ cells/well, incubated in complete McCoy's 5A for 24 hours, and then transfected with $50 \mathrm{nM}$ of siRNA. After transfection for 24,48 , and 72 hours, $10 \mu \mathrm{L}$ of the CCK-8 solution was added and incubated for 1.5 hours. The absorbance was measured at $450 \mathrm{~nm}$ using a microplate reader (as described earlier).

\section{Cell apoptosis assay}

An Annexin V-FITC apoptosis detection kit was utilized for evaluation of the percentage of apoptosis induced by Notch1 siRNA/DMAPA-chems nanoparticles $(\mathrm{N} / \mathrm{P}=100)$ at an siRNA dose of $50 \mathrm{nM}$, according to the protocol provided by the manufacturer. After transfection for 48 hours, floating and trypsinized adherent cells were gathered separately and rinsed with cold PBS three times. The cells were resuspended in $500 \mu \mathrm{L}$ of $1 \times$ Annexin $\mathrm{V}$ binding buffer containing $5 \mu \mathrm{L}$ Annexin V-FITC and $5 \mu \mathrm{L}$ propidium iodide and incubated in 
a darkroom at $4^{\circ} \mathrm{C}$ for 15 minutes. Analysis was immediately carried out by using a flow cytometer (described earlier).

\section{Statistical analysis}

All experiments were performed three or more times independently. Statistical analyses were carried out using one-way analysis of variance (GraphPad Prism 5, La Jolla, CA, USA) and Student's two-tailed $t$-test to analyze statistically significant differences between the data of various experimental groups. A value of $P<0.05$ was considered statistically significant.

\section{Results and discussion Preparation of the siRNA/DMAPA-chems nanoparticles}

Recently, novel cationic cholesterol derivatives have received attention as efficient nonviral gene delivery vectors due to their high gene transfection efficiency. ${ }^{29,30}$ In this study,
DMAPA-chems was successfully synthesized from cholesteryl, succinic anhydride, NHS, and DMAPA (data has been reported). DMAPA-chems and phospholipid S100 were used to produce liposomes by the film dispersion method. DMAPA-chems liposomes were added to siRNA solution at different N/P ratios for delivery of siRNA into SKOV3 cells in an effort to downregulate Notch1 gene expression (Figure 1).

\section{Particle size, zeta potential, and morphology assay}

The particle size and zeta potential of siRNA/DMAPA-chems complexes were measured using dynamic light scattering. As shown in Figure 2A, the zeta potentials were found to be positive when siRNA/DMAPA-chems complexes formed at N/P $>10$. Meanwhile, the zeta potentials of the siRNA/DMAPA-chems complexes increased in the range of +40 to $+50 \mathrm{mV}$ when their N/P ratios increased, which is attributed to the effective

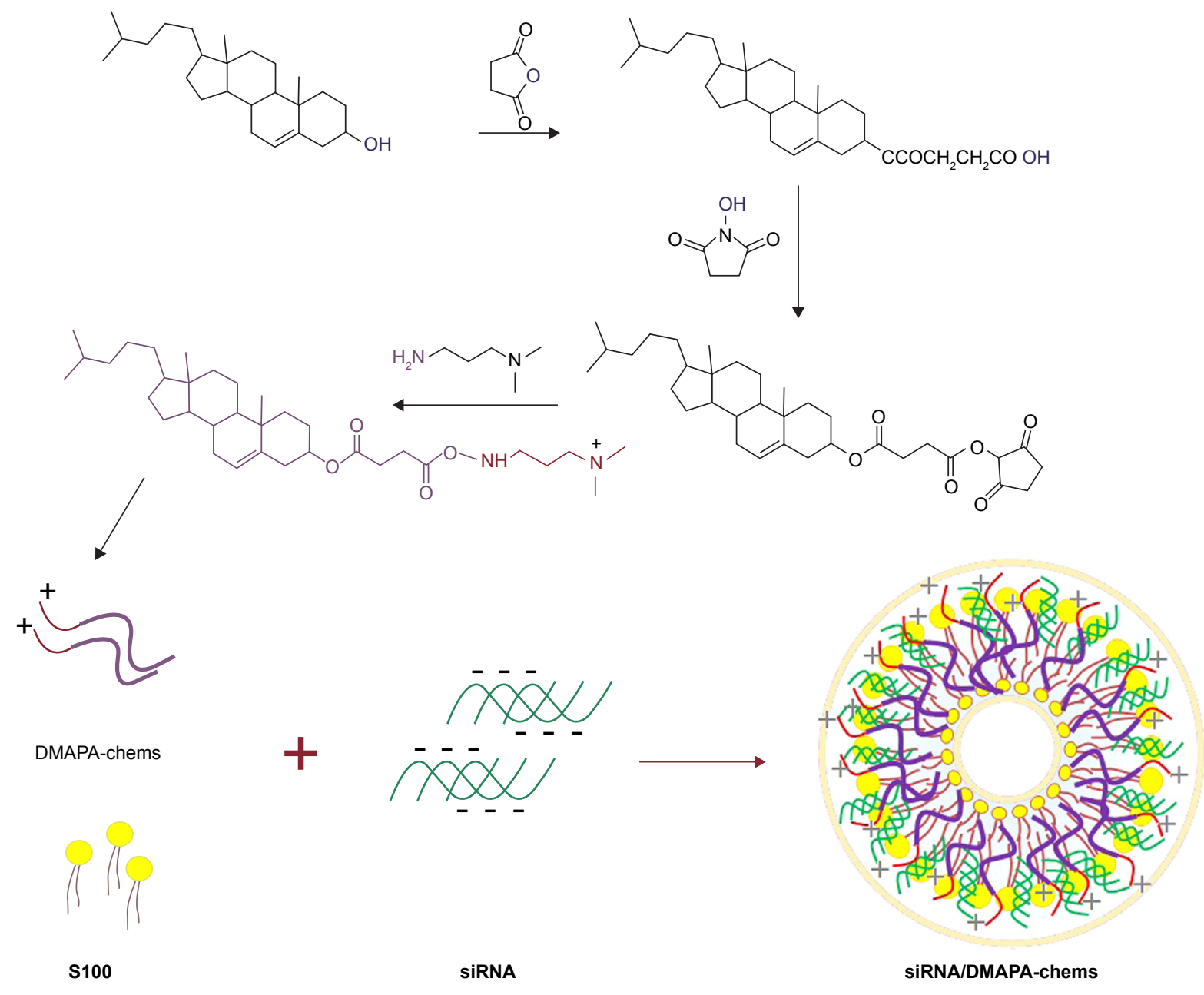

Figure I The synthetic mechanism of action of the cationic cholesterol derivative DMAPA-chems and the formulation of siRNA/DMAPA-chems complexes. Abbreviations: DMAPA-chems, $\mathrm{N}$-(cholesterylhemisuccinoyl-amino-3-propyl)- $\mathrm{N}, \mathrm{N}$-dimethylamine; siRNA, small interfering RNA. 
condensation of siRNA. The particle size of the siRNA/ DMAPA-chems complexes decreased following the N/P ratios increasing, but was still in the range of $100-200 \mathrm{~nm}$. This suggested that the complexes became progressively smaller the further the siRNA condensation processed.

The morphology of siRNA/DMAPA-chems complexes was investigated by transmission electron microscopy. As shown in Figure 2B, the complexes at an N/P ratio of 100 were observed to have a near-spherical shape. There was excellent cellular uptake owing to the positive zeta potential, ideal particle size, and spherical shape of the siRNA/ DMAPA-chems complexes.

\section{Agarose gel electrophoresis and stability analysis}

Agarose gel electrophoresis assay was utilized to observe the formation of the siRNA/cholesterol derivative nanoparticles, utilizing naked siRNA as a control. As shown in Figure 3A, the migration of siRNA was entirely arrested when the siRNA/DMAPA-chems N/P ratios exceeded 30. This indicated that cholesterol derivative DMAPA-chems liposomes could condense siRNA to the complexes, which probably aids in cellular uptake, elevates serum stability, and provides better protection from nuclease degradation.

Higher serum stability as well as lower cytotoxicity are pivotal for successful siRNA delivery, both in vitro and in vivo. ${ }^{31}$ The stability of siRNA/DMAPA-chems complexes $(\mathrm{N} / \mathrm{P}=100)$ was evaluated in 25\% FBS (Figure 3B). Naked siRNA control group was totally degraded within 24 hours of cultivation, while the migration of siRNA was still observed after siRNA/DMAPA-chems complexes treated with heparin solution were cultivated in $25 \%$ FBS. To analyze the stability

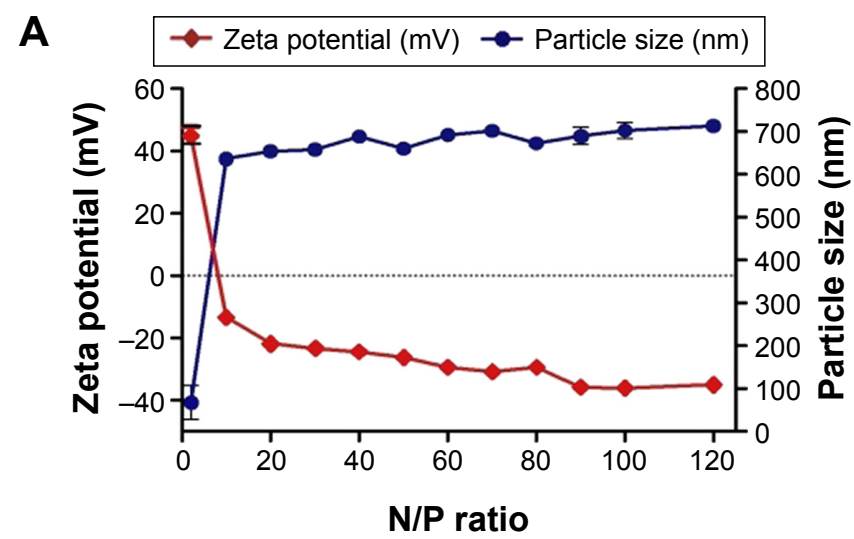

of siRNA/DMAPA-chems complexes (N/P =100) against nuclease, both naked siRNA and siRNA/DMAPA-chems complexes were exposed to $0.1 \mathrm{mg} / \mathrm{mL}$ RNase A at $37^{\circ} \mathrm{C}$ for 24 hours. The naked siRNA was completely degraded in RNase A solution within 0.5 hour, whereas the migration of siRNA could be still be observed after siRNA/DMAPAchems complexes were incubated in RNase A solution for 24 hours (Figure 3B). These results indicate that siRNA/ DMAPA-chems complexes exhibit an excellent protective effect against RNase A for siRNA in serum.

\section{Cytotoxicity assay}

CCK-8 assay was used to observe the cytotoxicity of the cationic cholesterol derivative DMAPA-chems liposome in SKOV3 cells. As shown in Figure 4A, cytotoxicity increased with increasing concentrations of DMAPA-chems liposome. At a DMAPA-chems liposome dose less than $80 \mu \mathrm{g} / \mathrm{mL}$, the derivative showed little cytotoxicity against SKOV3 cells. The viability of SKOV3 cells remained above $80 \%$. The low toxicity may be attributable to the lower molecular weight of the DMAPA-chems derivative as well as better biocompatibility of the cationic cholesterol derivative. ${ }^{20,32}$

A positive charge on nanoparticles is known to facilitate cellular uptake via a nonspecific electrostatic interaction between the positively charged nanoparticles and negatively charged cell membranes, but it is also considered a major cause of cytotoxicity. ${ }^{33}$ Thus, evaluating the cytotoxicity of the siRNA/ cholesterol derivative nanoparticles at different $\mathrm{N} / \mathrm{P}$ ratios was very important. As shown in Figure 4B, the siRNA/cholesterol derivative nanoparticles exhibited low cytotoxicity in the SKOV3 cells owing to the low cytotoxicity of the cholesterol derivative liposome. As the N/P ratio reached 120, cells treated

\section{B}

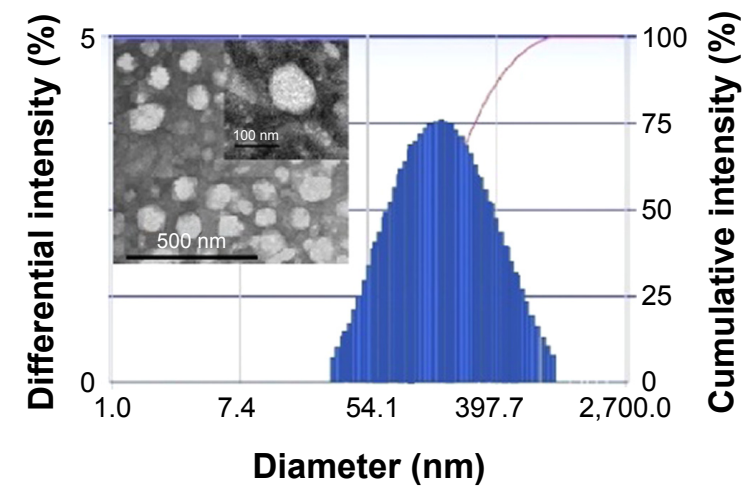

Figure 2 Particle size, zeta potential, and morphology assessment of siRNA/DMAPA-chems nanoparticles with different N/P ratios.

Notes: (A) Size distribution and zeta potential of siRNA/DMAPA-chems nanoparticles with different N/P ratios by DLS. (B) TEM images of siRNA/DMAPA-chems nanoparticles $(\mathrm{N} / \mathrm{P}=100)$ (scale bar $=500 \mathrm{~nm}, 80 \mathrm{~kW})$.

Abbreviations: DLS, dynamic light scattering; TEM, transmission electron microscope; siRNA, small interfering RNA; DMAPA-chems, N-(cholesterylhemisuccinoyl-amino3-propyl)-N, N-dimethylamine; N/P, nitrogen-to-phosphate. 




Figure 3 Agarose gel electrophoresis and stability analysis of siRNA/DMAPAchems complexes.

Notes: (A) Agarose gel electrophoresis retardation assay of siRNA/DMAPA-chems complexes at different N/P ratios at a fixed siRNA dose of I $\mu \mathrm{g}$. (B) Protection and release assay of siRNA/DMAPA-chems nanoparticles (N/P $=100$, at an siRNA dose of $\mathrm{I} \mu \mathrm{g})$ in $\mathrm{FBS}(25 \%)$ and $\mathrm{RNase} A$ solution $(0.1 \mathrm{mg} / \mathrm{mL})$, visualized on a $1.5 \%$ agarose gel. Abbreviations: siRNA, small interfering RNA; FBS, fetal bovine serum; DMAPAchems, $\mathrm{N}$-(cholesterylhemisuccinoyl-amino-3-propyl)- $\mathrm{N}, \mathrm{N}$-dimethylamine; N/P, nitrogen-to-phosphate. with the siRNA/cholesterol derivative nanoparticles for 72 hours maintained a relatively high viability of about $80 \%$.

\section{Quantification of cellular uptake}

Cellular uptake efficiency of gene/cationic polymers complexes is considered to depend on the surface properties and particle sizes. ${ }^{34,35}$ It has been reported that gene/cationic polymer complexes with positive charge and particle sizes of fifty to several hundred nanometers would be suitable for the endocytosis of complexes and for superior gene delivery. ${ }^{36}$ The zeta potentials and particle sizes of the prepared siRNA/ DMAPA-chems complexes $(\mathrm{N} / \mathrm{P}>20)$ were in the range of +40 to $+50 \mathrm{mV}$ and $100-200 \mathrm{~nm}$, respectively. These properties might facilitate the entry of the siRNA/DMAPAchems nanoparticles into the SKOV3 cells as expected. The SKOV3 cell uptake of the siRNA/DMAPA-chems nanoparticles was evaluated using flow cytometry and compared with the FAM-siRNA/Lipo 2000 complex as the positive control and the naked FAM-siRNA as the NC. The siRNA/ DMAPA-chems nanoparticles displayed significantly more fluorescent cells than the naked FAM-siRNA cells group, and an increase in the N/P ratio resulted in an increasing percentage of fluorescence in Figure 5A. At an N/P ratio of 100, the cellular uptake efficiency was $93.95 \% \pm 0.19 \%$, which was not significantly different from that observed for the siRNA/ Lipo-positive control group $(97.66 \% \pm 1.02 \%)$.

Cellular uptake was further detected by the accumulation and spread of green fluorescence of FAM-siRNA in the cytoplasm, as observed in the inverse fluorescent and confocal microscopy images (Figure 5B and C). The SKOV3 cells were incubated for 24 hours. After 6 hours of incubation with the FAM-siRNA/DMAPA-chems nanoparticles
A

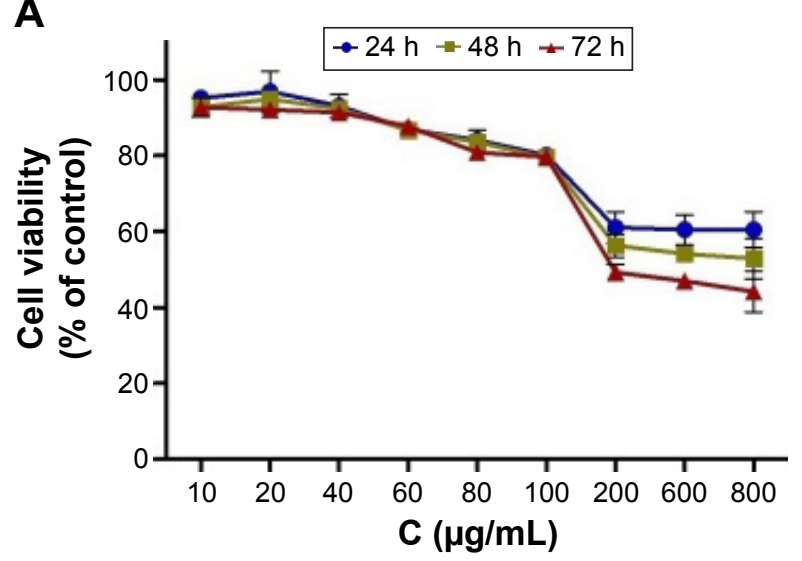

B



Figure 4 Cytotoxicity assay of DMAPA-chems liposomes and siRNA/DMAPA-chems complexes.

Notes: (A) Viability of SKOV3 cells incubated with the DMAPA-chems liposomes at various concentrations in the range from 10 to $800 \mu g / \mathrm{mL}$ ( $\mathrm{n}=3$ ) for 24,48 , and $72 \mathrm{~h}$. (B) Viability of SKOV3 cells incubated with the siRNA/DMAPA-chems complexes at various N/P ratios from I to I20 ( $\mathrm{n}=3)$ for 24,48 , and $72 \mathrm{~h}$.

Abbreviations: siRNA, small interfering RNA; DMAPA-chems, N-(cholesterylhemisuccinoyl-amino-3-propyl)-N, N-dimethylamine; N/P, nitrogen-to-phosphate. 


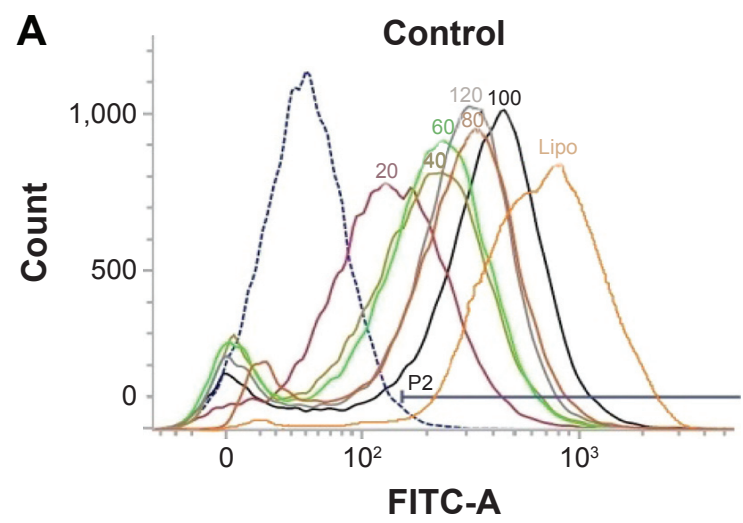

B

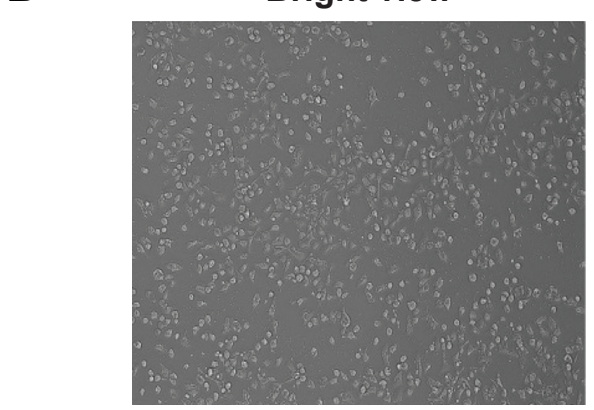

C

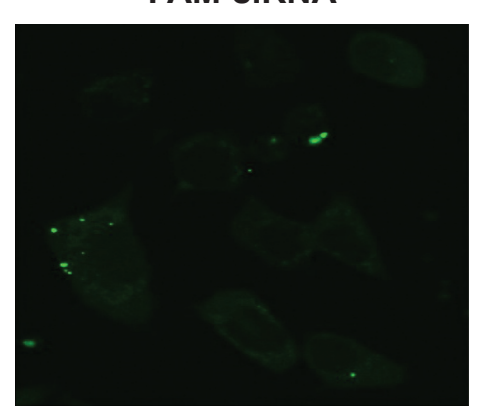



FAM-SIRNA

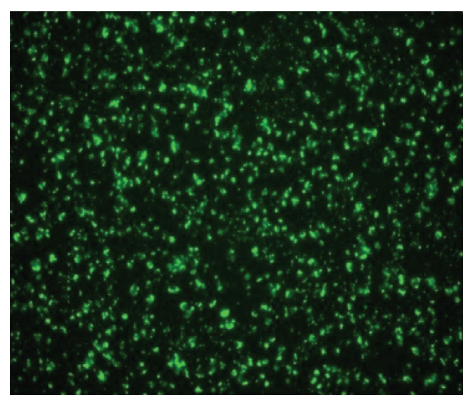

LysoTracker Blue DND


Merged

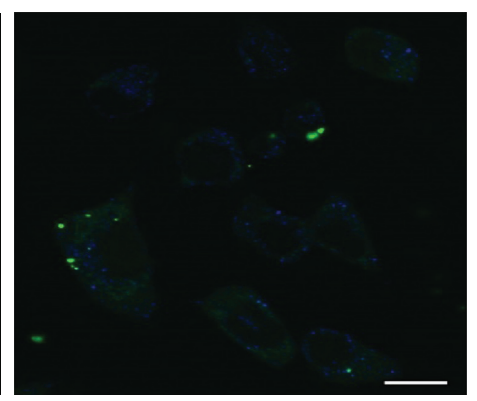

Figure 5 Quantification of cellular uptake analysis by flow cytometry, inverse fluorescence microscopy images, and laser scanning confocal microscopy images.

Notes: (A) Flow cytometry and quantitative determination of cell uptake efficiency of FAM-positive SKOV3 cells following incubation of SKOV3 cells with the different complexes at various N/P ratios from 20 to 120 at a FAM-siRNA final concentration of $50 \mathrm{nM}(\mathrm{n}=3)$ for 5 h. (B) Inverse fluorescent microscopic images $(\times 100)$ of SKOV3 cells treated with FAM-siRNA/DMAPA-chems $(\mathrm{N} / \mathrm{P}=100)$ at an siRNA dose of $50 \mathrm{nM}$ for $6 \mathrm{~h}$. (C) Endosomal/lysosomal escape of FAM-siRNA were imaged using a confocal laser scanning microscope. The SKOV3 cells were treated with FAM-siRNA/DMAPA-chems $(\mathrm{N} / \mathrm{P}=100)$ at an siRNA dose of $50 \mathrm{nM}$ for $6 \mathrm{~h}$ and then stained with LysoTracker Blue DND-22; scale bar is $5 \mu \mathrm{m}$.

Abbreviations: siRNA, small interfering RNA; Lipo, lipofectamine 2000; n, number; DMAPA-chems, N-(cholesterylhemisuccinoyl-amino-3-propyl)-N, N-dimethylamine; N/P, nitrogen-to-phosphate.

$(\mathrm{N} / \mathrm{P}=100)$, the spread of green fluorescence of FAM-siRNA in the cytoplasm indicated endocytosis and endosome escape of the siRNA/DMAPA-chems nanoparticles, which suggested the DMAPA-chems derivative has a good buffering capability.

\section{qRT-PCR analysis}

Notch1 gene inhibition by Notch1 siRNA/DMAPA-chems complexes $(\mathrm{N} / \mathrm{P}=80,100$, or 120) at an siRNA dose of $50 \mathrm{nM}$ as well as different siRNA doses $(30,50,80$, or $100 \mathrm{nM})$ of
Notch1 siRNA/DMAPA-chems complexes at an N/P ratio of 100 were evaluated at the mRNA level using qRT-PCR analysis. As shown in Figure 6A, at an siRNA dose of $50 \mathrm{nM}$, the expression efficiency of Notch 1 mRNA was significantly reduced by transfection with Notch1 siRNA/DMAPA-chems complexes with different $\mathrm{N} / \mathrm{P}$ ratios and was not significantly different from lipofectamine 2000. As shown in Figure 6B, at an N/P ratio of 100, Notch1 siRNA/DMAPA-chems complexes at an siRNA dose of $50 \mathrm{nM}$ displayed the most efficient target gene silencing with a Notch1 mRNA expression 
A

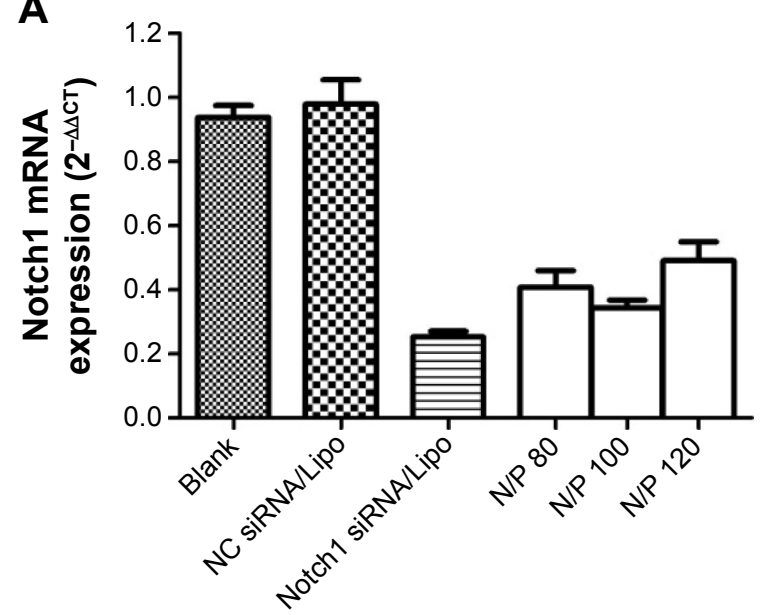

B

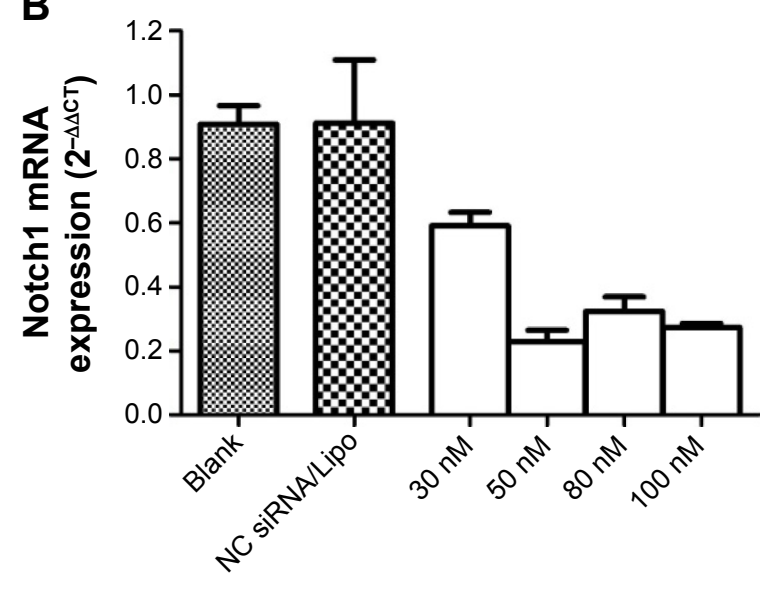

Figure 6 qRT-PCR analysis of NotchI mRNA expression.

Notes: (A) Notch I mRNA expression at various N/P ratios of siRNA/DMAPA-chems complexes at the same siRNA dose of $50 \mathrm{nM}$ after incubated for $48 \mathrm{~h}$. (B) Notch I mRNA expression analysis when cells were treated with different siRNA dosages of siRNA/DMAPA-chems complexes at the same N/P ratio of 100 after cultured for $48 \mathrm{~h}$. Values are presented as mean $\pm S D, n=4$.

Abbreviations: qRT-PCR, quantitative real-time polymerase chain reaction; RT-PCR, reverse transcription polymerase chain reaction; siRNA, small interfering RNA; $\mathrm{n}$, number; SD, standard deviation; N/P, nitrogen to phosphate ratios.

of $0.22 \pm 0.07$. These results indicate that the gene expression suppression efficacy relies heavily on cellular uptake, which is closely associated with the size, charge, and morphology of the siRNA/DMAPA-chems nanoparticles.

\section{Western blot analysis}

Western blot assay was used to measure the protein expression level of Notch1 in SKOV3 cells. As shown in Figure 7, SKOV3 cells incubated with the Notch1 siRNA/DMAPA-chems nanoparticles had lower levels of Notch1 protein with increasing incubation times, as compared with the NC group $(P<0.05)$. At 72 hours, the Notch1 protein expression efficiency decreased to
$26.47 \% \pm 6.23 \%$. There was no significant difference between the Notch1 siRNA/DMAPA-chems nanoparticles group and the positive control group $(P>0.05)$.

\section{Cell proliferation and apoptosis assay} SKOV3 cells were transfected with Notch1 siRNA/DMAPAchems nanoparticles at various N/P ratios (80, 100, and120) and NC siRNA/DMAPA-chems nanoparticles (N/P =80, 100, and 120) were examined 24 hours later to confirm that the cell line was stable. As shown in Figure 8A, the cell growth was significantly inhibited at 24, 48, and 72 hours after treatment with Notch1 siRNA/DMAPA-chems nanoparticles
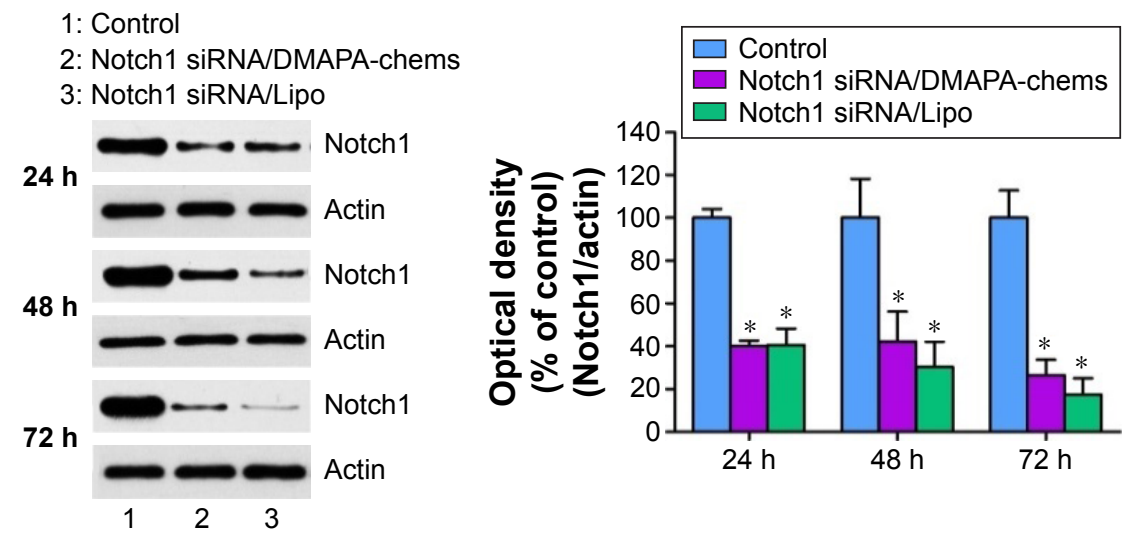

Figure 7 Western blot analysis of SKOV3 cells.

Notes: Suppression of the protein expression of the Notchl gene in SKOV3 cells evaluated by Western blot analysis after transfection with siRNA/DMAPA-chems complexes at an N/P ratio of 100 and an siRNA dose of $50 \mathrm{nM}$ for 24,48 , and $72 \mathrm{~h}$. The data are presented as mean $\pm S D$ ( $n=4$, $* P<0.01$, compared with the control group and the Notch I siRNA/DMAPA-chems or Notch I siRNA/Lipo group).

Abbreviations: siRNA, small interfering RNA; n, number; DMAPA-chems, $N$-(cholesterylhemisuccinoyl-amino-3-propyl)-N, N-dimethylamine; SD, standard deviation; N/P, nitrogen-to-phosphate. 

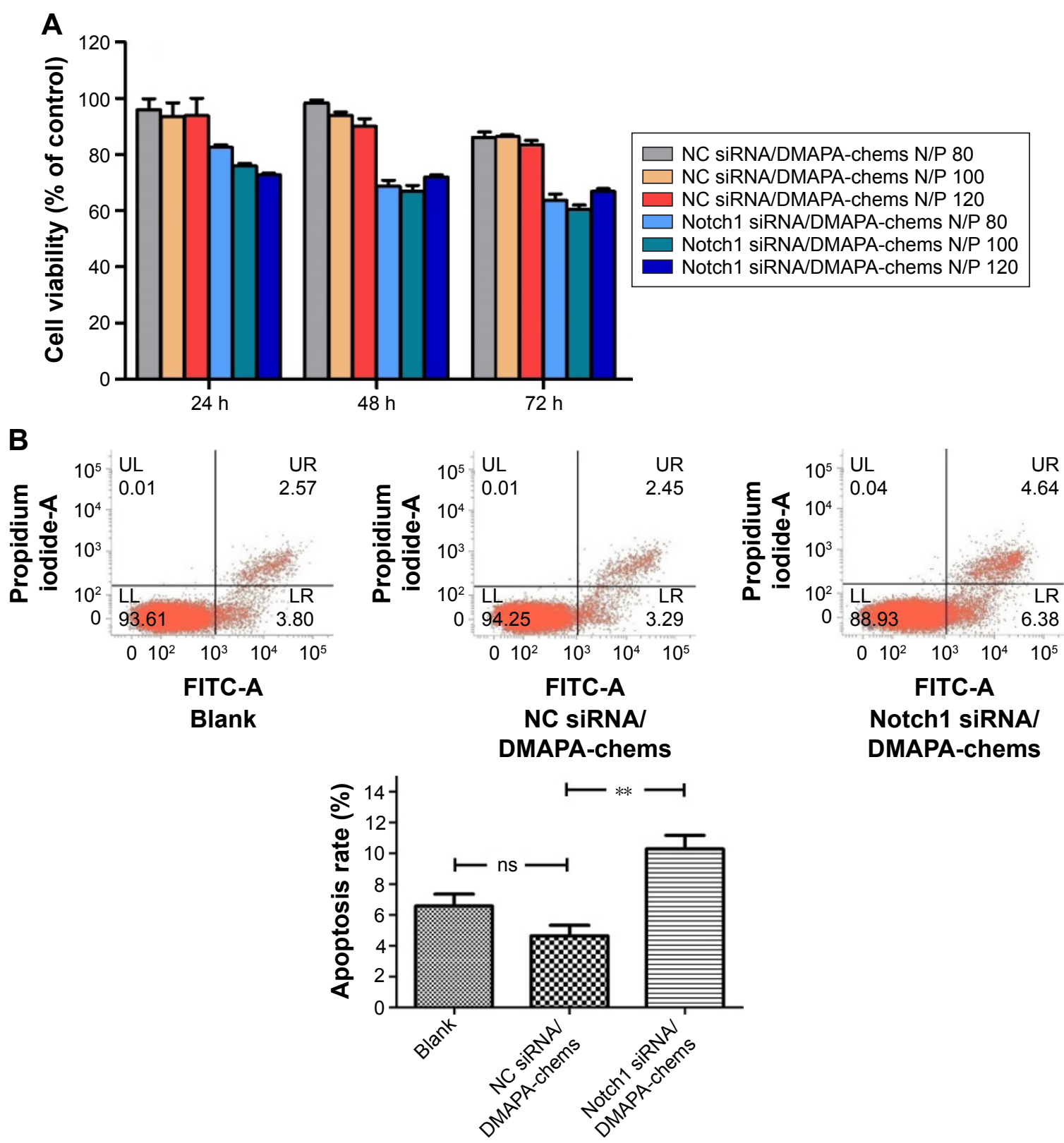

Figure 8 Proliferation and apoptosis assay of SKOV3 cells.

Notes: (A) The viability of SKOV3 cells after transfection with NC siRNA/DMAPA-chems complexes (N/P =80, 100, and I20) and Notch I siRNA /DMAPA-chems complexes (N/P =80, 100, and I20) with $50 \mathrm{nM}$ of siRNA. The results represent the mean $\pm S D(n=5)$. (B) Annexin V-FITC apoptosis detection kit was used to measure the percentage of apoptosis induced by NC siRNA/DMAPA-chems complexes $(\mathrm{N} / \mathrm{P}=100)$ and Notch I siRNA /DMAPA-chems complexes $(\mathrm{N} / \mathrm{P}=100)$ with $50 \mathrm{nM}$ of siRNA. All the data are presented as mean $\pm S D, n=4$. $* * P<0.01$.

Abbreviations: NC, negative control; siRNA, small interfering RNA; n, number; SD, standard deviation; DMAPA-chems, N-(cholesterylhemisuccinoyl-amino-3-propyl)-N, $\mathrm{N}$-dimethylamine; N/P, nitrogen-to-phosphate; FITC, fluorescein isothiocyanate; ns, no significant differences; UL, upper left; UR, upper right; LL, lower left; LR, lower right.

(N/P =80, 100 and 120), while SKOV3 cells treated with NC siRNA/DMAPA-chems nanoparticles (N/P =80, 100, and 120) showed no statistical significant change in growth, indicating that siRNA/DMAPA-chems nanoparticles have minimal toxic effects on the cells.

We have reported that imbalances between cell proliferation and the rate of apoptosis allow cancer to grow uncontrolled. ${ }^{23}$ Thus, we analyzed the effects of NC siRNA/DMAPA-chems nanoparticles and Notch1 siRNA/DMAPA-chems nanoparticles at the same N/P ratio $(\mathrm{N} / \mathrm{P}=100)$. As shown in Figure 8B, treatment with Notch1 siRNA/DMAPA-chems nanoparticles increased the percentage of apoptotic cells $(P<0.01)$, whereas treatment with NC siRNA/DMAPAchems nanoparticles did not show any significant differences compared with the blank cell group. The data suggested that Notch1 siRNA/DMAPA-chems nanoparticles could influence 
both growth and survival of SKOV3 cells. Furthermore, these findings were similar to other reports that indicated that blocking active Notch1 leads to apoptosis of cells and inhibition of cell proliferation..$^{16,37-39}$

\section{Conclusion}

In this study, we utilized a novel cationic cholesterol derivative-based liposome, DMAPA-chems, for efficient delivery of siRNA into human SKOV3 ovarian cancer cells to inhibit Notch1 gene expression. The siRNA/DMAPA-chems nanoparticles with a size of $100-200 \mathrm{~nm}$, a positive charge of $40-50 \mathrm{mV}$, and a near-spherical morphology are very stable in serum and RNase solution. Furthermore, the siRNA/DMAPAchems nanoparticles showed low toxicity against SKOV3 cells and had excellent cellular uptake and gene silencing efficiency. This study also demonstrated that siRNA/DMAPA-chems nanoparticles could inhibit the growth of SKOV3 cells and promote apoptosis. These in vitro results imply that the cationic cholesterol derivative-based DMAPA-chems liposomes may be a good vector for siRNA delivery in vivo.

\section{Acknowledgments}

This work was financially supported by National Natural Science Foundation of China, General Program (nos 81173000 , 81102396, 81201606, and 81200428) and Natural Science Foundation of Zhejiang Province, People's Republic of China (nos LY14H300005 and LY16H160025).

\section{Disclosure}

The authors report no conflicts of interest in this work.

\section{References}

1. McLachlan J, Banerjee S. Olaparib for the treatment of epithelial ovarian cancer. Expert Opin Pharmacother. 2016;17(7):995-1003.

2. Spirtos NM, Gross GM, Freddo JL, Ballon SC. Cytoreductive surgery in advanced epithelial cancer of the ovary: the impact of aortic and pelvic lymphadenectomy. Gynecol Oncol. 1995;56(3):345-352.

3. Wei Z, Wang Y, Yu X, Zhang S. Identification and characterization of stem cells in an ovarian cancer cell line and examination their drug resistance. Zhonghua Fu Chan Ke Za Zhi. 2015;50(6):452-457.

4. Fan Q, Cai Q, Xu Y. FOXM1 is a downstream target of LPA and YAP oncogenic signaling pathways in high grade serous ovarian cancer. Oncotarget. 2015;6(29):27688-27699.

5. Du P, Zhang X, Liu H, Chen L. Lentivirus-Mediated RNAi silencing targeting ERCC1 reverses cisplatin resistance in cisplatin-resistant ovarian carcinoma cell line. DNA Cell Biol. 2015;34(7):497-502.

6. Peart T, Ramos Valdes Y, Correa RJ, et al. Intact LKB1 activity is required for survival of dormant ovarian cancer spheroids. Oncotarget. 2015;6(26):22424-22438.

7. Kim HJ, Kim A, Miyata K, Kataoka K. Recent progress in development of siRNA delivery vehicles for cancer therapy. Adv Drug Deliv Rev. 2016;104:61-77.

8. Zhang L, Zheng W, Tang R, Wang N, Zhang W, Jiang X. Gene regulation with carbon-based siRNA conjugates for cancer therapy. Biomaterials. 2016;104:269-278.
9. Huang YP, Hung CM, Hsu YC, et al. Suppression of breast cancer cell migration by small interfering RNA delivered by polyethylenimine functionalized graphene oxide. Nanoscale Res Lett. 2016;11(1):247.

10. Yamamoto S, Schulze KL, Bellen HJ. Introduction to Notch signaling. Methods Mol Biol. 2014;1187:1-14.

11. Nicolas M, Wolfer A, Raj K, et al. Notch1 functions as a tumor suppressor in mouse skin. Nat Genet. 2003;33(3):416-421.

12. Yuan $\mathrm{X}, \mathrm{Wu} \mathrm{H}, \mathrm{Han} \mathrm{N}$, et al. Notch signaling and EMT in non-small cell lung cancer: biological significance and therapeutic application. J Hematol Oncol. 2014;7:87.

13. Miele L. Notch signaling. Clin Cancer Res. 2006;12(4):1074-1079.

14. Rose SL, Kunnimalaiyaan M, Drenzek J, Seiler N. Notch 1 signaling is active in ovarian cancer. Gynecol Oncol. 2010;117(1):130-133.

15. Wang M, Wu L, Wang L, Xin X. Down-regulation of Notch1 by gammasecretase inhibition contributes to cell growth inhibition and apoptosis in ovarian cancer cells A2780. Biochem Biophys Res Commun. 2010;393(1): 144-149.

16. Wang M, Lv Y, Yi K, Wang L, Xin X, Wang Y. Down-regulation of Notch1 by gamma-secretase inhibitor contributes to growth inhibition and apoptosis of ovarian cancer cells A2780. Cancer Res Prev Treat. 2014;957-961.

17. Wang H, Huang X, Zhang J, et al. The expression of VEGF and D114/ Notch pathway molecules in ovarian cancer. Clin Chim Acta. 2014;436: 243-248.

18. Haussecker D. Current issues of RNAi therapeutics delivery and development. J Control Release. 2014;195:49-54.

19. Mantha N, Das SK, Das NG. RNAi-based therapies for Huntington's disease: delivery challenges and opportunities. Ther Deliv. 2012;3(9): 1061-1076.

20. He P, Hagiwara K, Chong H, Yu HH, Ito Y. Low-molecular-weight polyethyleneimine grafted polythiophene for efficient siRNA delivery. Biomed Res Int. 2015;2015:406389.

21. Yao Y, Su Z, Liang Y, Zhang N. pH-Sensitive carboxymethyl chitosanmodified cationic liposomes for sorafenib and siRNA co-delivery. Int J Nanomedicine. 2015;10:6185-6197.

22. Zhu H, Dong C, Dong H, et al. Cleavable PEGylation and hydrophobic histidylation of polylysine for siRNA delivery and tumor gene therapy. ACS Appl Mater Interfaces. 2014;6(13):10393-10407.

23. Zhao Y, Zheng C, Zhang L, Chen Y, Ye Y, Zhao M. Knockdown of STAT3 expression in SKOV3 cells by biodegradable siRNA-PLGA/CSO conjugate micelles. Colloids Surf B Biointerfaces. 2015;127:155-163.

24. Ding W, Hattori Y, Higashiyama K, Maitani Y. Hydroxyethylated cationic cholesterol derivatives in liposome vectors promote gene expression in the lung. Int J Pharm. 2008;354(1-2):196-203.

25. Han SE, Kang H, Shim GY, et al. Novel cationic cholesterol derivativebased liposomes for serum-enhanced delivery of siRNA. Int J Pharm. 2008;353(1-2):260-269.

26. Liu Z, Gong H, Zeng R, et al. Efficient delivery of NF-kB siRNA to human retinal pigment epithelial cells with hyperbranched cationic polysaccharide derivative-based nanoparticles. Int $J$ Nanomedicine. 2015; 10:2735-2749

27. Hattori Y, Nakamura T, Ohno H, Fujii N, Maitani Y. siRNA delivery into tumor cells by lipid-based nanoparticles composed of hydroxyethylated cholesteryl triamine. Int J Pharm. 2013;443(1-2):221-229.

28. Wang $\mathrm{W}$, Zheng HL, Chen LN. Synthesis and characterization of $\mathrm{N}-\left(\mathrm{N}^{\prime}\right.$, $\mathrm{N}^{\prime}$-dimethyl) propyl succinic mono-cholesteryl mono-amide as gene carrier. Chinese Pharmaceut J. 2015;12:1032-1037.

29. Jubeli E, Maginty AB, Khalique NA, et al. Next generation macrocyclic and acyclic cationic lipids for gene transfer: Synthesis and in vitro evaluation. Bioorg Med Chem. 2015;23(19):6364-6378.

30. Vulugundam G, Kumar K, Kondaiah P, Bhattacharya S. Efficacious redox-responsive gene delivery in serum by ferrocenylated monomeric and dimeric cationic cholesterols. Org Biomol Chem. 2015;13(14): 4310-4320.

31. Yang C, Zhang C, Zhao Z, Zhu T, Yang B. Fighting against kidney diseases with small interfering RNA: opportunities and challenges. J Transl Med. 2015;13(1):39. 
32. Ju J, Huan ML, Wan N, Qiu H, Zhou SY, Zhang BL. Novel cholesterolbased cationic lipids as transfecting agents of DNA for efficient gene delivery. Int J Mol Sci. 2015;16(3):5666-5681.

33. Khatri N, Baradia D, Vhora I, Rathi M, Misra A. Development and characterization of siRNA Lipoplexes: effect of different lipids, in vitro evaluation in cancerous cell lines and in vivo toxicity study. AAPS PharmSciTech. 2014;15(6):1630-1643.

34. Shan Y, Panday N, Myoung Y, et al. Scanning ion conductance microscopic study for cellular uptake of cationic conjugated polymer nanoparticles. Macromol Biosci. 2016;16(4):599-607.

35. He Y, Park K. Effects of the microparticle shape on cellular uptake. Mol Pharm. 2016;13(7):2164-2171.

36. Gan Q, Wang T, Cochrane C, McCarron P. Modulation of surface charge, particle size and morphological properties of chitosan-TPP nanoparticles intended for gene delivery. Colloids Surf B Biointerfaces. 2005;44(2-3):65-73.
37. Kim MJ, Park JS, Lee SJ, et al. Notch1 targeting siRNA delivery nanoparticles for rheumatoid arthritis therapy. J Control Release. 2015; 216:140-148.

38. Zhou Y, Chen Q, Qin R, Zhang K, Li H. MicroRNA-449a reduces cell survival and enhances cisplatin-induced cytotoxicity via downregulation of NOTCH1 in ovarian cancer cells. Tumour Biol. 2014;35(12): 12369-12378

39. Dai MY, Fang F, Zou Y, et al. Down regulation of Notch1 induces apoptosis and inhibits cell proliferation and metastasis in laryngeal squamous cell carcinoma. Oncol Rep. 2015;34(6):3111-3119.

\section{Publish your work in this journal}

The International Journal of Nanomedicine is an international, peerreviewed journal focusing on the application of nanotechnology in diagnostics, therapeutics, and drug delivery systems throughout the biomedical field. This journal is indexed on PubMed Central,

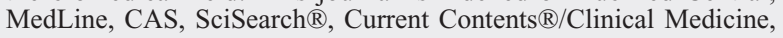

Journal Citation Reports/Science Edition, EMBase, Scopus and the Elsevier Bibliographic databases. The manuscript management system is completely online and includes a very quick and fair peer-review system, which is all easy to use. Visit http://www.dovepress.com/ testimonials.php to read real quotes from published authors. 